\title{
Pseudopath semiclassical approximation to transport through open quantum billiards: Dyson equation for diffractive scattering
}

\author{
Christoph Stampfer, ${ }^{*}$ Stefan Rotter, and Joachim Burgdörfer \\ Institute for Theoretical Physics, Vienna University of Technology, Wiedner Hauptstraße 8-10/136, 1040 Vienna, Austria, \\ European Union \\ Ludger Wirtz \\ Institute for Electronics, Microelectronics and Nanotechnology, Bô̂te Postale 60069, 59652 Villeneuve d'Ascq Cedex, France, \\ European Union
}

(Received 6 April 2005; published 30 September 2005)

\begin{abstract}
We present a semiclassical theory for transport through open billiards of arbitrary convex shape that includes diffractively scattered paths at the lead openings. Starting from a Dyson equation for the semiclassical Green's function we develop a diagrammatic expansion that allows a systematic summation over classical and pseudopaths, the latter consisting of classical paths joined by diffractive scatterings ("kinks"). This renders the inclusion of an exponentially proliferating number of pseudopath combinations numerically tractable for both regular and chaotic billiards. For a circular billiard and the Bunimovich stadium the path sum leads to a good agreement with the quantum path length power spectrum up to long path length. Furthermore, we find excellent numerical agreement with experimental studies of quantum scattering in microwave billiards where pseudopaths provide a significant contribution.
\end{abstract}

DOI: 10.1103/PhysRevE.72.036223

PACS number(s): 05.45.Mt, 73.23.Ad, 73.50.Bk, 03.65.Sq

\section{INTRODUCTION}

Semiclassical approximations are among the most useful tools in describing and analyzing ballistic transport in mesoscopic systems. On a fundamental level, semiclassical techniques allow one to build a bridge between classical and quantum mechanics: the classical paths carry an amplitude which reflects the geometric stability of the orbits and a phase that contains the classical action and accounts for quantum interference [1-3].

Ballistic transport through billiards has been studied extensively in the last decade [4-23] and a variety of semiclassical approximations [7-15,24-26] have been introduced in order to provide a qualitative and, in part, also a quantitative description of these systems. In particular, universal conductance fluctuations (UCFs) and the "weak localization" (WL) have been studied [14] in order to delineate characteristic differences in the quantum transport of classically chaotic and integrable billiards. Very recently, quantum shot noise [27] in ballistic cavities that are either chaotic [28,29], regular [30], or display a mixed phase space [31] has been used as a probe of the quantum-to-classical and chaotic-to-regular crossover [26,28-34].

The approach of the (semi)classical limit of ballistic quantum transport is both conceptually as well as numerically nontrivial as it represents, generically, a multiscale problem. The two-dimensional quantum billiard (or quantum dot, see Fig. 1) is characterized by an area $A$ or linear dimension $D=\sqrt{A}$. The quantum wires (or leads) to which the billiard is

\footnotetext{
*Present address: Chair of Micro and Nanosystems, Swiss Federal Institute of Technology Zurich (ETH Zurich), Tannenstr. 3, 8092 Zurich, Switzerland.
}

attached have the width $d$. In order to reach sufficiently long dwell times such that differences between transiently regular and chaotic motion become important, the relation $d / D \ll 1$ should hold. To approach the semiclassical limit for the motion inside the billiard requires $\lambda_{D} \ll D\left(\lambda_{D}\right.$ de Broglie wave length) or equivalently $k D \gg 2 \pi$. Furthermore, if the (disorder) potential inside the dot varies over a length scale $a_{P}$, we should require $\lambda_{D} \ll a_{P}$ for a semiclassical approximation to hold. These conditions pertaining to the dot are necessary but not sufficient. Since the scattering $(S)$ matrix maps asymptotic scattering states onto each other, also the entrance and exit channel states in the quantum wire should reach their classical limit $\lambda_{D} \ll d$ or $k d \gg 2 \pi$. The latter limit is virtually impossible to reach, neither experimentally for quantum dots $[28,35,36]$ or microwave billiards [37] nor numerically [38]. We will therefore focus in the following on the "intermediate" semiclassical regime pertaining to the interior of the billiard $\lambda_{D} \ll D$ with convex hard-walled boundaries such that quantum diffraction in the interior can be neglected, with the understanding, however, that quantum

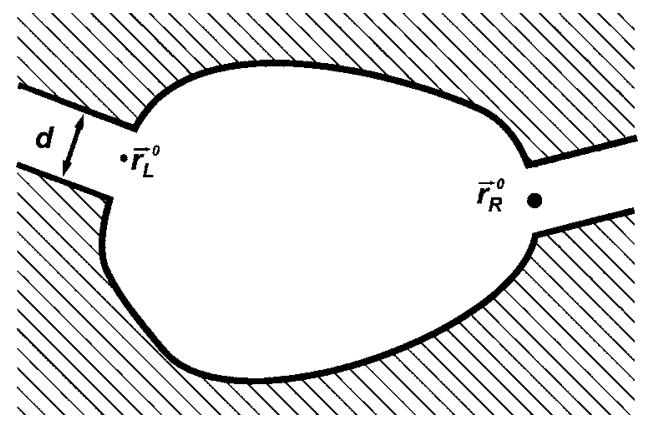

FIG. 1. An open arbitrarily convex-shaped billiard with two narrow leads of equal width $d$ : left $(L)$ and right $(R)$. 
effects due to the coupling to the asymptotic quantum wires have to be taken into account. Accordingly, the term "semiclassical approximations" refers in the following to approximations to the constant-energy Green's function for propagation in the interior of the $\operatorname{dot} G\left(\vec{r}, \vec{r}^{\prime}, k\right)$ by an approximate semiclassical limit, $G^{\mathrm{SC}}$ to be discussed below. The projections of $G$ onto asymptotic scattering states with transverse quantum numbers $m_{L}\left(m_{R}\right)$ yield the amplitudes for transmission $T_{m_{L}, m_{R}}$ from the entrance (left) to the exit (right) lead and reflections $R_{m_{L}, m_{L}^{\prime}}$. Standard semiclassical approximations to $G$ face several fundamental difficulties $[10,11,14,18,25,39]$ : among many others, unitarity is violated with discrepancies in some cases as large as the conductance fluctuations the theory attempts to describe $[11,12]$. Likewise, the anticorrelation $\delta|T|^{2}=-\delta|R|^{2}$ between transmission fluctuations $\delta|T|^{2}$ and the corresponding fluctuations in the reflection $\delta|R|^{2}$ as a function of the wave number $k$ is broken. Also, the "weak localization" effect is considerably overestimated [16,39]. These difficulties are due to the fact that hard-walled billiards possess "sharp edges" at the entrance and exit leads even though the interior of the dot features a smooth (in the present case, a constant) potential. At these sharp edges the contacts to the quantum wires feature spatial variations of the potential where the length scale $a_{P}$ approaches zero. Consequently the semiclassical limit $\lambda_{D} / a_{P} \ll 1$ cannot be reached, no matter how small $\lambda_{D}$ (or large $k$ ) is. In other words, the quantum properties of the leads influence also the semiclassical dynamics in the interior. This observation is the starting point for diffractive corrections such as the Kirchhoff diffraction [7] or Fraunhofer diffraction [10].

We have recently developed a pseudopath semiclassical approximation (PSCA) [15] with pseudopaths that result from spawning of classical paths due to diffractive (i.e., nongeometric) reflections in the lead mouths (or point contacts). Pseudopaths play an essential role when incorporating indeterministic features into the semiclassical description of transport. Their existence has also recently been pointed out in Ref. [40], although no explicit numerical investigations were performed. While classical trajectories are either ejected through the exit lead contributing to $T$ or return back to the entrance lead contributing to $R$, a quantum wave packet will do both. Pseudopaths interconnect otherwise disjunct subsets of classical paths that exit either through the left or right lead. The lack of this coupling is responsible for violation of the anticorrelation of transmission and reflection fluctuations, $\delta T^{2} \neq-\delta R^{2}$, in standard semiclassical approximation (SCA). Likewise, the standard SC approximation is expected to fail for quantum shot noise [27] that is a signature of this quantum indeterminism. For the special case of the rectangular shaped dot with lead openings placed at the midpoints, summation of exponentially proliferating pseudopaths could be accomplished by tracing trajectories in an extended zone scheme $[15,41]$. For arbitrarily shaped billiards and, in particular, chaotic billiards where already classical trajectories proliferate exponentially, systematic inclusion of paths and pseudopaths up to the same length is considerably more complicated. In the following we address this problem within the framework of a semiclassical Dyson equation. We present a diagrammatic expansion that allows a systematic summation of classical path and pseudopath contributions. Applications to the circular billiard (as a prototypical regular system) and to the Bunimovich stadium billiard (as the prototype system for chaotic scattering) show good agreement with the numerically calculated exact quantum path length spectrum. We furthermore apply the PSCA to recent experimental studies of scattering in microwave billiards in which pseudopath contributions could be experimentally identified.

The structure of the paper is as follows. In Sec. II we briefly review the standard semiclassical approximation and previous attempts to include diffraction effects. The pseudopath semiclassical approximation for arbitrarily convex shaped billiards will be presented in Sec. III employing a semiclassical version of the Dyson equation. We develop a diagrammatic expansion of $G$ in terms of paths and pseudopaths. Its evaluation is numerically facilitated by an algebraic matrix representation that reduces path summations up to infinite order to a sequence of matrix multiplications and inversions, as discussed in Sec. IV. Numerical results and comparison with the full quantum results as well as microwave experiments are given in Sec. V, followed by a short summary and outlook onto future applications in Sec. VI. Details on the Fraunhofer diffraction approximation are given in the Appendix.

\section{STANDARD SEMICLASSICAL APPROXIMATION}

The conductance of a ballistic two-terminal system (as depicted in Fig. 1) is determined by the scattering amplitudes $T_{m}$ through the Landauer formula [42]

$$
g(k)=\frac{2 e^{2}}{h}\left(\sum_{m_{L}=1}^{M} \sum_{m_{R}=1}^{M}\left|T_{m_{R}, m_{L}}(k)\right|^{2}\right),
$$

where $M$ is the number of open modes in the leads (quantum wires) and $T_{m_{R}, m_{L}}(k)$ are the transmission amplitudes from the $m_{L}$ th mode in the entrance lead [referred to in the following as left $(L)$ lead] to the $m_{R}$ th mode in the exit lead [referred to as the right $(R)$ lead]. In the following we choose local coordinate systems $\left(x_{i}, y_{i}\right), i=L, R$ for the leads, where $x_{i}$ denotes the longitudinal and $y_{i}$ the transverse direction of lead $i$. For simplicity we use the coding $(L, R)$ for the entrance and exit channels throughout this publication, irrespective of the actual location at which the leads are attached. The projection of the retarded Green's propagator onto the transverse wave functions $\phi_{m_{L}}\left(y_{L}\right)$ and $\phi_{m_{R}}\left(y_{R}\right)$ of incoming and outgoing modes [14] serves as starting point for most semiclassical theories which approximate transmission amplitudes from mode $m_{L}$ to mode $m_{R}$ :

$$
\begin{aligned}
T_{m_{L}, m_{R}}(k)= & -i \sqrt{k_{x_{R}, m_{R}} k_{m_{L}, x_{L}}} \int d y_{R} \int d y_{L} \phi_{m_{R}}^{*}\left(y_{R}\right) \\
& \times G\left(x_{R}, y_{R}, x_{L}, y_{L}, k\right) \phi_{m_{L}}\left(y_{L}\right) .
\end{aligned}
$$

Here and in the following we use atomic units $\left(\hbar=|e|=m_{e}\right.$ $=1)$. The transverse wave-functions $\phi_{m}(y)$ are given for zero magnetic field $(B=0)$ by 


$$
\phi_{m}(y)=\sqrt{\frac{2}{d}} \begin{cases}\cos \left(\frac{m \pi}{d} y\right), & m \text { odd }, \\ \sin \left(\frac{m \pi}{d} y\right), & m \text { even },\end{cases}
$$

where $d$ denotes the width of the leads. We assume, for simplicity, that the two leads have identical width. The scattering state in the lead is the product of the transverse lead wave function $\phi_{m}(y)$ and a plane wave in the longitudinal direction with wave vector $k_{x, m}=\sqrt{k^{2}-|m \pi / d|^{2}}$. Both leads have a total of $(m=1, \ldots, M)$ open (i.e., transmitting) modes. Analogously, the amplitude for reflection from the incoming mode $m_{L}$ into a different mode $m_{L}^{\prime}$ in the same quantum wire is given by

$$
\begin{aligned}
R_{m_{L}, m_{L}^{\prime}}= & \delta_{m_{L}, m_{L}^{\prime}}-i \sqrt{k_{x_{L}, m_{L}^{\prime}} k_{m_{L}, x_{L}}} \int d y_{L}^{\prime} \int d y_{L} \\
& \times \phi_{m_{L}^{\prime}}^{*}\left(y_{L}^{\prime}\right) G\left(x_{L}^{\prime}, y_{L}^{\prime}, x_{L}, y_{L}, k\right) \phi_{m_{L}}\left(y_{L}\right) .
\end{aligned}
$$

Equations (2.2) and (2.4) can be written in terms of flux normalized projectors onto the left (right) lead or point contact $\left(x_{L, R}^{0}, y_{L, R}^{0}\right)$ with matrix elements

$$
\begin{aligned}
\left\langle\vec{r}\left|P_{L}\right| \vec{r}^{\prime}\right\rangle= & \sqrt{\left|k_{x_{L}}\right|\left|k_{x_{L}}^{\prime}\right|} \delta\left(\vec{r}-\vec{r}^{\prime}\right) \delta\left(x-x_{L}^{0}\right) \\
& \times\left[\Theta\left(y-y_{L}^{0}+d / 2\right)-\Theta\left(y-y_{L}^{0}-d / 2\right)\right], \\
\left\langle\vec{r}\left|P_{R}\right| \vec{r}^{\prime}\right\rangle= & \sqrt{\left|k_{x_{R}}\right|\left|k_{x_{R}}^{\prime}\right|} \delta\left(\vec{r}-\vec{r}^{\prime}\right) \delta\left(x-x_{R}^{0}\right) \\
& \times\left[\Theta\left(y-y_{R}^{0}+d / 2\right)-\Theta\left(y-y_{R}^{0}-d / 2\right)\right]
\end{aligned}
$$

as

$$
\begin{gathered}
T_{m_{R}, m_{L}}(k)=-i\left\langle m_{R}\left|P_{R} G(k) P_{L}\right| m_{L}\right\rangle, \\
R_{m_{L}^{\prime}, m_{L}}(k)=\delta_{m_{L}, m_{L}^{\prime}}-i\left\langle m_{L}^{\prime}\left|P_{L} G(k) P_{L}\right| m_{L}\right\rangle .
\end{gathered}
$$

The factor $\left|k_{x_{L}}\right|\left(\left|k_{x_{L}}^{\prime}\right|\right)$ denotes the longitudinal component of the momentum of the incoming (outgoing) wave function at the left lead.

The semiclassical approximation to the scattering amplitudes is obtained by approximating the Green's function $G\left(\vec{r}_{2}, \vec{r}_{1}, k\right)$ in Eq. (2.2) by the semiclassical Green's propagator $G^{\mathrm{SC}}\left(\vec{r}_{2}, \vec{r}_{1}, k\right)$. The standard semiclassical Green's propagator $G^{\mathrm{SC}}\left(\vec{r}_{2}, \vec{r}_{1}, k\right)$, the Fourier-Laplace transform of the van Vleck propagator evaluated in stationary phase approximation (SPA), describes the probability amplitude for propagation from $\vec{r}_{1}$ to $\vec{r}_{2}$ at a fixed energy, $E=k^{2} / 2$. It can be expressed in terms of a sum over all classical paths of energy $E$ (or, equivalently, wave vector $k$ ) connecting these two points [1],

$$
\begin{aligned}
G^{\mathrm{SC}}\left(\vec{r}_{2}, \vec{r}_{1}, k\right)= & \sum_{\alpha: \vec{r}_{1} \rightarrow \vec{r}_{2}} G_{\alpha}^{\mathrm{SC}}=\sum_{\alpha: \vec{r}_{1} \rightarrow \vec{r}_{2}} \frac{\left|D_{\alpha}\left(\vec{r}_{2}, \vec{r}_{1}, k\right)\right|^{1 / 2}}{(2 \pi i)^{1 / 2}} \\
& \times \exp \left[i\left(S_{\alpha}\left(\vec{r}_{2}, \vec{r}_{1}, k\right)-\frac{\pi}{2} \mu_{\alpha}\right)\right] .
\end{aligned}
$$

Here, $S_{\alpha}\left(\vec{r}_{2}, \vec{r}_{1}, k\right)=k L_{\alpha}$ is the action of the path $\alpha$ of length $L_{\alpha} . \quad D_{\alpha}\left(\vec{r}_{2}, \vec{r}_{1}, k\right)$ is the classical deflection factor [1] which describes the stability of the paths and $\mu_{\alpha}$ denotes the Maslov index of the path $\alpha$. In line with the semiclassical approximation, the double integrals in Eqs. (2.2) and (2.4) are frequently evaluated in stationary phase approximation. Physically this means that the paths are entering and exiting the cavity only with the discrete angles $\theta_{m}=\arcsin [m \pi /(d k)]$ due to the quantization of the transverse momentum in the leads. For completeness we mention at this point that the path sum in Eq. (2.8) also contains, as a subset, those orbit pairs described in Ref. [25] which yield a weak-localization correction beyond the diagonal approximation.

Several strategies have been proposed to introduce diffractive effects in order to quantitatively improve the semiclassical theory for transport through open quantum billiards. A straightforward way is to eliminate the SPA for the double integral in Eq. (2.4). Expanding the action in the semiclassical Green's function Eq. (2.8) to first order in the transverse coordinate, the integral takes the form of a Fraunhofer diffraction integral and can be evaluated analytically [10]. On this level of approximation, diffraction effects are thereby automatically included, however, only upon entering and exiting the cavity, not during propagation inside the cavity. Schwieters et al. [7] employed Kirchhoff diffraction theory to calculate the diffractional weight of paths entering and exiting the billiard. In addition, they introduced the concept of "ghost paths": paths that are specularly reflected at the lead opening due to diffractive effects. The proper use of a diffractive weight for classical paths allowed the quantitative determination of the peak heights in the power spectra of the transmission and reflection amplitudes $[7,10]$ - at least for short path lengths. Ghost orbits could account for some of the peaks that were missing in the semiclassical spectra [7]. Several deficiencies remained, however, unresolved: unitarity of the semiclassical $S$ matrix is, typically, violated; the weak localization peak is significantly underestimated, and the semiclassical pathlength $(l)$ spectrum [17]

$$
P_{m^{\prime}, m}^{\mathrm{SC}}(l)=\left|\int d k e^{i k l} T_{m^{\prime}, m}^{\mathrm{SC}}(k)\right|^{2},
$$

fails to account for all the peaks and overestimates the corresponding quantum pathlength spectrum for large $l$. For the special case of a rectangular billiard we could recently demonstrate [15] that a systematic inclusion of pseudopaths within the pseudopath semiclassical approximation (PSCA) has the potential to overcome these deficiencies. In the following we derive the PSCA for an arbitrary convex billiard from a semiclassical Dyson equation and investigate its properties numerically. 


\section{DYSON EQUATION FOR THE PSCA}

Starting point is a semiclassical version of the Dyson equation for the Green's function where the standard semiclassical Green's function $G^{\mathrm{SC}}$ plays the role of the unperturbed Green's function and the diffractive scatterings at the lead openings (or point contacts) are the perturbation. Accordingly, we have

$$
G^{\mathrm{PSC}}=G^{\mathrm{SC}}+G^{\mathrm{SC}} V G^{\mathrm{PSC}},
$$

where the perturbation "potential" $V$ is given in terms of the projectors Eqs. (2.5) and (2.6) as

$$
V=P_{L}+P_{R}
$$

Iterative solution by summation

$$
G^{\mathrm{PSC}}=G^{\mathrm{SC}} \sum_{i=0}^{\infty}\left(V G^{\mathrm{SC}}\right)^{i}=G^{\mathrm{SC}} \sum_{i=0}^{\infty}\left[\left(P_{L}+P_{R}\right) G^{\mathrm{SC}}\right]^{i}
$$

includes diffractive scatterings into the $G^{\mathrm{PSC}}$ to all orders.

The key to the multiple diffractive scattering expansion is that within the semiclassical expansion each projection operator onto the $L$ and $R$ point contacts selects classical trajectories emanating from or ending up at the leads and at the same time spawns new generations of classical trajectories.

Noting that $G^{\mathrm{PSC}}$ will only be evaluated in the domain of $P_{L}$ or $P_{R}$, Eq. (3.3) can be reorganized in terms of a $2 \times 2$ matrix Dyson equation. We decompose $G^{\mathrm{SC}}$ as follows:

$$
\begin{gathered}
P_{L} G^{\mathrm{SC}} P_{L} \Rightarrow G_{L L}^{\mathrm{SC}}=\sum_{\alpha_{L L}} G_{\alpha_{L L}}^{\mathrm{SC}} \\
P_{L} G^{\mathrm{SC}} P_{R} \Rightarrow G_{L R}^{\mathrm{SC}}=\sum_{\alpha_{L R}} G_{\alpha_{L R}}^{\mathrm{SC}}, \\
P_{R} G^{\mathrm{SC}} P_{L} \Rightarrow G_{R L}^{\mathrm{SC}}=\sum_{\alpha_{R L}} G_{\alpha_{R L}}^{\mathrm{SC}}, \\
P_{R} G^{\mathrm{SC}} P_{R} \Rightarrow G_{R R}^{\mathrm{SC}}=\sum_{\alpha_{R R}} G_{\alpha_{R R}}^{\mathrm{SC}} .
\end{gathered}
$$

In Eq. (3.4) the index $\alpha_{i j}$ refers to paths that emanate from the point contact $j(j=L, R)$ and end up at point contact $i(i=L, R)$. Each of the four disjunct subsets of classical paths is, in general, infinite. In the following we denote the truncated number of trajectories of the corresponding class by $N_{i j}$. Each $G_{i j}^{\mathrm{SC}}$ containing a large finite number $N_{i j}$ or an infinite number of trajectories is diagrammatically represented by a double line, each contribution of an individual trajectory by a single line (Fig. 2). The trajectories emanate or end on vertices representing the $L$ or $R$ point contacts.

Equation (3.1) now becomes

$$
\begin{aligned}
\left(\begin{array}{cc}
G_{L L}^{\mathrm{PSC}} & G_{L R}^{\mathrm{PSC}} \\
G_{R L}^{\mathrm{PSC}} & G_{R R}^{\mathrm{PSC}}
\end{array}\right)= & \left(\begin{array}{cc}
G_{L L}^{\mathrm{SC}} & G_{L R}^{\mathrm{SC}} \\
G_{R L}^{\mathrm{SC}} & G_{R R}^{\mathrm{SC}}
\end{array}\right)+\left(\begin{array}{cc}
G_{L L}^{\mathrm{SC}} & G_{L R}^{\mathrm{SC}} \\
G_{R L}^{\mathrm{SC}} & G_{R R}^{\mathrm{SC}}
\end{array}\right) \times\left(\begin{array}{cc}
P_{L} & 0 \\
0 & P_{R}
\end{array}\right) \\
& \times\left(\begin{array}{ll}
G_{L L}^{\mathrm{PSC}} & G_{L R}^{\mathrm{PSC}} \\
G_{R L}^{\mathrm{PSC}} & G_{R R}^{\mathrm{PSC}}
\end{array}\right) .
\end{aligned}
$$

Its solution $G^{\mathrm{PSC}}$ denoted by thick solid lines can now be

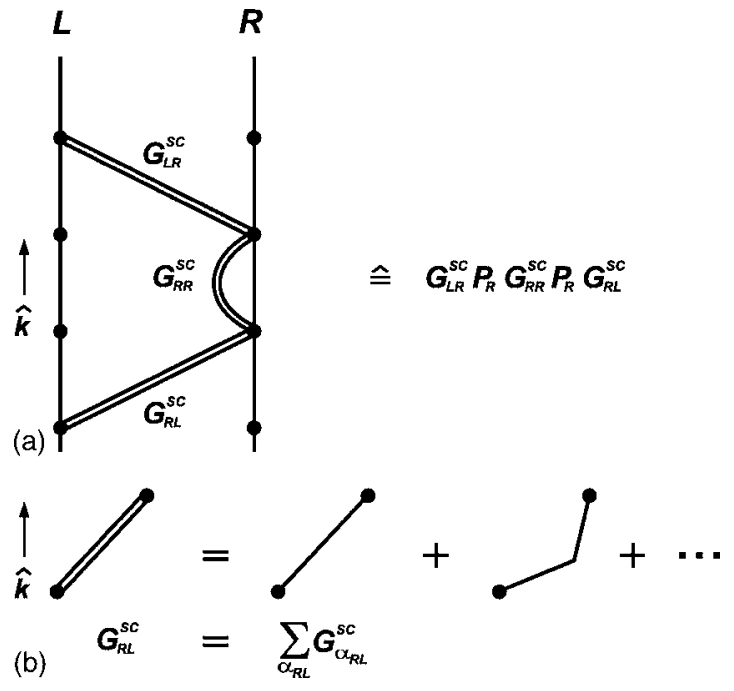

FIG. 2. Diagramatic expansion of paths. Double line: $G_{i j}^{\mathrm{SC}}$, single line: contribution of individual classical trajectories $G_{\alpha_{i j}}^{\mathrm{SC}}$, dots: replica of point contacts along the propagation direction $\hat{k}$ ("time"). (a) Typical term appearing in the expansion of $G^{\mathrm{PSC}}$. (b) Expansion of $G_{i j}^{\mathrm{SC}}$ in terms of classical path contributions.

diagrammatically represented (Fig. 3) as a sum over all pseudopaths that result from the couplings of classical-path Green's functions by successive diffractive scatterings at point contacts. Only pseudopath combinations (or diagrams) contribute that are connected at vertices $L$ or $R$.

Equation (3.5) can be formally solved by matrix inversion

$$
\begin{aligned}
\left(\begin{array}{cc}
G_{L L}^{\mathrm{PSC}} & G_{L R}^{\mathrm{PSC}} \\
G_{R L}^{\mathrm{PSC}} & G_{R R}^{\mathrm{PSC}}
\end{array}\right)= & \left(\begin{array}{cc}
1-G_{L L}^{\mathrm{SC}} P_{L} & -G_{L R}^{\mathrm{SC}} P_{R} \\
-G_{R L}^{\mathrm{SC}} P_{L} & 1-G_{R R}^{\mathrm{SC}} P_{R}
\end{array}\right)^{-1} \\
& \times\left(\begin{array}{cc}
G_{L L}^{\mathrm{SC}} & G_{L R}^{\mathrm{SC}} \\
G_{R L}^{\mathrm{SC}} & G_{R R}^{\mathrm{SC}}
\end{array}\right)
\end{aligned}
$$

resulting in a sum over (up to) infinitely long pseudo paths with (up to) an infinite number of diffractive scatterings. Note that the number of classical paths between two diffractive "kinks" and their lengths may reach infinity as well. The handling of this double limit plays an important role in the numerical implementation as destructive interference of pseudopaths and classical paths of comparable length must be properly taken into account. The evaluation of Eq. (3.5) and (3.6) is not straightforward as each operator product in Eq. (3.3) contains multiple two-dimensional integrals over $\mathrm{R}^{2}$. For illustrative purposes we explicitly give the first-

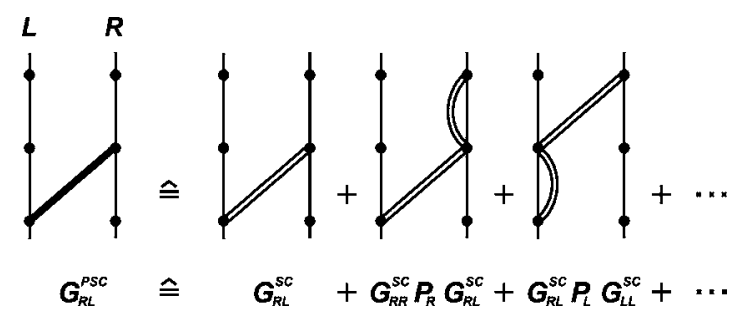

FIG. 3. Thick solid line: $G^{\mathrm{PSC}}$; first terms of the expansion of the Dyson equation for $G_{R L}^{\mathrm{PSC}}$. 
correction term $\left(G^{\mathrm{SC}} P_{L} G^{\mathrm{SC}}+G^{\mathrm{SC}} P_{R} G^{\mathrm{SC}}\right)$ of Eq. (3.5). We have to calculate the double integral, e.g.,

$$
G_{L R}^{\mathrm{SC}} P_{R} G_{R L}^{\mathrm{SC}}=\int_{\mathbb{R}^{2}} d^{2} r^{\prime} \int_{\mathbb{R}^{2}} d^{2} r G_{L R}^{\mathrm{SC}}\left(\vec{r}_{2}, \vec{r}^{\prime}\right) P_{R}\left(\vec{r}^{\prime}, \vec{r}\right) G_{R L}^{\mathrm{SC}}\left(\vec{r}, \vec{r}_{1}\right),
$$

where $\vec{r}_{2}\left(\vec{r}_{1}\right)$ are both located on the left point contact. The double integral reduces to a one-dimensional integral along the lead openings due to the $\delta$-functions in the projector Eq. (2.6),

$$
\begin{aligned}
G_{L R}^{\mathrm{SC}} P_{R} G_{R L}^{\mathrm{SC}}= & \int_{-d / 2}^{d / 2} d y \sqrt{\left|k_{x_{R}}\right|\left|k_{x_{R}}^{\prime}\right|} \\
& \times G_{L R}^{\mathrm{SC}}\left(\vec{r}_{2}, x_{R}^{0}, y_{R}^{0}+y\right) G_{R L}^{\mathrm{SC}}\left(x_{R}^{0}, y_{R}^{0}+y, \vec{r}_{1}\right) .
\end{aligned}
$$

Inserting the expression for the semiclassical Green's function Eq. (2.8), and using the abbreviation $\vec{r}_{R}=\left(x_{R}^{0}, y_{R}^{0}+y\right)$ we obtain

$$
\begin{aligned}
G_{L R}^{\mathrm{SC}} P_{R} G_{R L}^{\mathrm{SC}}= & -\sum_{\alpha_{R L}} \sum_{\alpha_{L R}} \int_{-d / 2}^{d / 2} d y \sqrt{\left|k_{x_{R}}\right|\left|k_{x_{R}}^{\prime}\right|} G_{\alpha_{L R}}^{\mathrm{SC}}\left(\vec{r}_{2}, \vec{r}_{R}\right) G_{\alpha_{R L}}^{\mathrm{SC}}\left(\vec{r}_{R}, \vec{r}_{1}\right)=-\frac{1}{2 \pi i} \sum_{\alpha_{R L}} \sum_{\alpha_{L R}}\left|\bar{D}_{\alpha_{R L}}\right|\left|\bar{D}_{\alpha_{L R}}\right| \\
& \times \int_{-d / 2}^{d / 2} d y \sqrt{\left|k_{x_{R}}\right|\left|k_{x_{R}}^{\prime}\right|} \exp \left[i k\left[L_{\alpha_{L R}}\left(\vec{r}_{1}, \vec{r}_{R}\right)+L_{\alpha_{R L}}\left(\vec{r}_{R}, \vec{r}_{2}\right)\right]-i \frac{\pi}{2}\left(\mu_{\alpha_{R L}}+\mu_{\alpha_{L R}}\right)\right],
\end{aligned}
$$

where we have assumed that the classical deflection factors $D_{\alpha}$ are smooth functions over the range of the lead mouth and can be approximated by their value $\bar{D}_{\alpha}$ at the center. In Eq. (3.9) $\alpha_{R L}$ denotes paths connecting the left with the right lead $(L \rightarrow R)$ while $\alpha_{L R}$ represents paths $(R \rightarrow L)$. In order to solve the integral in Eq. (3.9) analytically, we expand the path length $L_{\alpha}$ of the classical paths $\alpha$ leading from point $\vec{r}_{i}$ to the lead mouth $\vec{r}_{R}$ (here we set $y_{R}=0$ for simplicity and only consider regular billiards):

$$
L_{\alpha_{R L}}\left(\vec{r}_{R}, \vec{r}_{i}\right)=L_{\alpha_{R L}}+\sin \left(\theta_{\alpha_{R L}}\right) y+\frac{\cos \left(\theta_{\alpha_{R L}}\right)}{L_{\alpha_{R L}}} y^{2} \cdots,
$$

where $L_{\alpha_{R L}}=L_{\alpha_{R L}}\left(\vec{r}_{R}, \vec{r}_{i}\right)$ is the length of the path that reaches the center of the lead mouth.

Taking into account only the first-order correction in the lateral displacement, the integral in Eq. (3.9) takes the form of a Fraunhofer diffraction integral and can be solved analytically $[10,15]$ (see the Appendix also),

$$
\begin{aligned}
I= & \int_{-d / 2}^{d / 2} d y \sqrt{\left|k_{x_{R}}(y)\right|\left|k_{x_{R}}^{\prime}(y)\right|} \times \exp \left\{i k\left[L_{\alpha_{L R}}(y)+L_{\alpha_{R L}}(y)\right]\right\} \\
\approx & k \sqrt{\cos \left(\theta_{\alpha_{L R}}\right) \cos \left(\theta_{\alpha_{R L}}^{\prime}\right)} \int_{-d / 2}^{d / 2} d y \exp \left\{i k\left[L_{\alpha_{L R}}+L_{\alpha_{R L}}\right]\right. \\
& \left.+i k\left[\sin \left(\theta_{\alpha_{L R}}\right)+\sin \left(\theta_{\alpha_{R L}}^{\prime}\right)\right] y\right\} \\
= & \exp \left[i k\left(L_{\alpha_{L R}}+L_{\alpha_{R L}}\right)\right] r\left(\theta_{\alpha_{L R}}, \theta_{\alpha_{R L}}^{\prime}, k\right),
\end{aligned}
$$

where $\theta_{\alpha_{R L}}^{\prime}$ is the ending angle of the incoming path and $\theta_{\alpha_{L R}}$ is the starting angle of the exiting path. Furthermore, $r\left(\theta_{\alpha_{L R}}, \theta_{\alpha_{R L}}^{\prime}, k\right)$ corresponds to the Fraunhofer reflection coefficient at an open lead [15]:

$$
\begin{aligned}
r\left(\theta_{\alpha_{L R}}, \theta_{\alpha_{R L}}^{\prime}, k\right)= & 2 \sqrt{\cos \left(\theta_{\alpha_{L R}}\right) \cos \left(\theta_{\alpha_{R L}}^{\prime}\right)} \\
& \times\left\{\frac{\sin \left[\frac{k d}{2}\left(\sin \theta_{\alpha_{L R}}+\sin \theta_{\alpha_{R L}}^{\prime}\right)\right]}{\sin \theta_{\alpha_{L R}}+\sin \theta_{\alpha_{R L}}^{\prime}}\right\} .
\end{aligned}
$$

Finally, this first order correction term in Eq. (3.7) can be written explicitly as

$$
G_{L R}^{\mathrm{SC}} P_{R} G_{R L}^{\mathrm{SC}}=\sum_{\alpha_{L R}} \sum_{\alpha_{R L}} G_{\alpha_{L R}}^{\mathrm{SC}} r\left(\theta_{\alpha_{L R}}, \theta_{\alpha_{R L}}^{\prime}, k\right) G_{\alpha_{R L}}^{\mathrm{SC}} .
$$

Note that classical paths contributing to $G^{\mathrm{SC}}$ do not incorporate the finite width of the point contact since we take the limit $d \rightarrow 0$ in the simulation of classical paths. Instead they are specularly reflected at the open lead mouth $R$ as if there were a hard wall. Such a path will interfere with the pseudopath that has almost the same length and topology but experiences a nonspecular, i.e., diffractive reflection at the same point contact.

All higher-order corrections are evaluated analogously. Each additional vertex connecting two $G_{\mathrm{SC}}$ 's gives rise to an additional Fraunhofer integral with an interior reflection amplitude $r\left(\theta^{\prime}, \theta\right)$.

\section{MATRIX REPRESENTATION OF PSEUDOPATH SUM}

A numerical representation of the Dyson equation [Eqs. (3.5) and (3.6)] requires the truncation of the number of contributing paths in each of the four basic Green's functions [Eq. (3.4)] to large but finite numbers. Let $N_{L L}$ denote the number of paths leading from $\vec{r}_{L}^{0}$ back to $\vec{r}_{L}^{0}(L \rightarrow L)$. Accordingly, $N_{R R}$ is the number of paths of the class $(R \rightarrow R), N_{R L}$ the number for $(L \rightarrow R)$, and $N_{L R}$ the num- 
ber for $(R \rightarrow L)$. Note that $N_{R L}=N_{L R}$ for systems with time reversal symmetry. The total number of contributing paths is $N=N_{L L}+N_{R R}+N_{L R}+N_{L R}$. In order to numerically solve the semiclassical Dyson Equation (3.5), we write the semiclassical Green's function $G^{\mathrm{SC}}$ as a diagonal $N \times N$ matrix where each diagonal matrix element represents the contribution of one particular classical path. Distinguishing the four different subclasses of paths, the matrix can be written in the following form:

$$
\underline{G}^{\mathrm{SC}}(k)=\left(\begin{array}{cccc}
\underline{G}_{L L}^{\mathrm{SC}}(k) & & & 0 \\
& \underline{G}_{R L}^{\mathrm{SC}}(k) & & \\
& & \underline{G}_{R R}^{\mathrm{SC}}(k) & \\
0 & & & \underline{\underline{G}}_{L R}^{\mathrm{SC}}(k)
\end{array}\right),
$$

where the matrix elements of the submatrices are given by, e.g.,

$$
\underline{G}_{\alpha_{L L}}^{\mathrm{SC}}(k)=\frac{\left|D_{\alpha_{L L}}\right|^{1 / 2}}{(2 \pi i)^{1 / 2}} \exp \left[i\left(k L_{\alpha_{L L}}-\frac{\pi}{2} \mu_{\alpha_{L L}}\right)\right],
$$

and $\alpha_{L L}=1, \ldots, N_{L L}$. The point to be emphasized is that the reduction of the $(2 \times 2)$ block structure of the matrix Dyson integral equation [Eq. (3.5)] into a purely algebraic equation requires an extension to a $4 \times 4$ block structure [Eq. (4.1)]. The latter is the minimum size required to accomodate the eight independent block matrices representing the vertices [see Eq. (4.4) below]. Due to diffractive coupling at the lead mouths, the matrix $\underline{G}^{\mathrm{PSC}}$ that represents the Green's function in the pseudopath semiclassical approximation also contains nondiagonal matrix elements. A specific matrix element $\left(\alpha^{\prime}, \alpha\right)$ consists of a sum of all path combinations that contain the classical path $\alpha$ as first segment and the classical paths $\alpha^{\prime}$ as last segment.

The coupling between different classical paths is represented by the nondiagonal vertex matrix $\underline{\underline{r}}(k)$. The matrix elements are given - within the Fraunhofer diffraction approximation - by the reflection amplitudes [Eq. (3.12)]. Since $r\left(\theta, \theta^{\prime}, k\right)$ depends on both the angle of emission $\theta$ of the new path and the angle of incidence $\theta^{\prime}$ of the previous path, 16 block matrices would result. However, due to the restriction imposed by the projections $P_{L}$ and $P_{R}$ that the endpoint $(L$ or $R)$ of the incoming trajectory must agree with the starting point of the outgoing trajectory, effectively only eight block matrices can contribute. These can be characterized by the starting points and endpoints of classical trajectories at the vertex. The block matrices resulting from the projection $P_{L}$ are $\underline{\underline{r}}_{L L, L L}, \underline{\underline{r}}_{L L, L R}, \underline{\underline{r}}_{R L, L L}$, and $\underline{\underline{r}}_{R L, L R}$. Analogously, from the vertex $P_{R}$, we get $\underline{\underline{r}}_{R R, R R}, \underline{\underline{r}}_{R R, R L}, \underline{\underline{r}}_{L R, R R}$, and $\underline{\underline{r}}_{L R, R L}$. For instance, $\underline{\underline{r}}_{L L, L R}$ is a $N_{L L} \times N_{L R}$ matrix with the elements

$$
\underline{\underline{r}}_{\alpha_{L L}, \alpha_{L R}^{\prime}}(k)=\left\{r\left(\theta_{\alpha_{L L}}, \theta_{\alpha_{L R}}^{\prime}, k\right)\right\} .
$$

The full matrix is

$$
\underline{\underline{r}}(k)=\left(\begin{array}{cccc}
\underline{\underline{r}}_{L L, L L}(k) & 0 & 0 & \underline{\underline{r}}_{L L, L R}(k) \\
\underline{\underline{r}}_{R L, L L}(k) & 0 & 0 & \underline{\underline{r}}_{R L, L R}(k) \\
0 & \underline{\underline{r}}_{R R, R L}(k) & \underline{\underline{r}}_{R R, R R}(k) & 0 \\
0 & \underline{\underline{r}}_{L R, R L}(k) & \underline{\underline{r}}_{L R, R R}(k) & 0
\end{array}\right) .
$$

For billiards of arbitrary shape and positions of the leads, no further reductions are possible. Only for structures with discrete geometric symmetries $(L \leftrightarrow R)$ or time-reversal symmetry, the number of nonequivalent trajectories and thus of independent amplitudes $r\left(\theta, \theta^{\prime}, k\right)$ is reduced.

With Eqs. (4.3) and (4.4), the Dyson equation Eq. (3.5) can now be written as an algebraic matrix equation

$$
\begin{gathered}
\underline{\underline{G}}^{\mathrm{PSC}}(k)=\underline{\underline{G}}^{\mathrm{SC}}(k) \sum_{i=0}^{\infty}\left[\underline{\underline{r}}(k) \underline{\underline{G}}^{\mathrm{SC}}(k)\right]^{i} \\
=\underline{\underline{G}}^{\mathrm{SC}}(k)\left[1-\underline{\underline{r}}(k) \underline{\underline{G}}^{\mathrm{SC}}(k)\right]^{-1} .
\end{gathered}
$$

Equation (4.5b) represents the "exact" summation over pseudopaths with up to an infinite number of diffractive scatterings. The accuracy of the result is, however, limited by the fact that we can only take into account a finite number $N$ of classical paths.

Finally, calculation of the $S$ matrix elements requires the projection of $G^{\mathrm{PSC}}$ onto the asymptotic scattering states in the left and right quantum wire [Eqs. (2.7a) and (2.7b)]. Following the same line of reasoning as in Eqs. (3.9)-(3.12), the projections $P_{L}$ and $P_{R}$ give rise to a transmission amplitude in Fraunhofer diffraction approximation. The transmission amplitude from incoming mode $m$ to a classical path $\alpha$ inside the cavity with launching angle $\theta_{\alpha}$ is given by [15]

$$
\begin{aligned}
t_{m}\left(\theta_{\alpha}, k\right)= & \sqrt{\frac{2 \cos \theta_{\alpha}}{k d}}\left[\frac{\sin \left[\left(k \sin \theta_{\alpha}+\frac{m \pi}{d}\right) \frac{d}{2}\right]}{\sin \theta_{\alpha}+\frac{m \pi}{k d}}\right. \\
& +\frac{\sin \left[\left(k \sin \theta_{\alpha}-\frac{m \pi}{d}\right) \frac{d}{2}\right]}{\left.\sin \theta_{\alpha}-\frac{m \pi}{k d}\right]} .
\end{aligned}
$$

Likewise, the transmission amplitude for a trajectory $\alpha$ approaching the point contact with angle $\theta_{\alpha}$ to exit in mode $m$ is also given by Eq. (4.6).

With Eq. (4.6) we form now amplitude matrices to map the asymptotic scattering states onto the $N \times N$ representation of $G^{\mathrm{PSC}}$. The $N \times 2 M$ matrix for the incoming state, where $M$ is the number of open transverse modes $\left(m_{L, R}=1, \ldots, M\right)$, is given by 


$$
\stackrel{A^{\mathrm{in}}}{=}(k)=\left(\begin{array}{cc}
t_{m_{L}}\left(\theta_{\alpha_{L L}}, k\right) & 0 \\
t_{m_{L}}\left(\theta_{\alpha_{R L}}, k\right) & 0 \\
0 & t_{m_{R}}\left(\theta_{\alpha_{R R}}, k\right) \\
0 & t_{m_{R}}\left(\theta_{\alpha_{L R}}, k\right)
\end{array}\right) .
$$

The corresponding projection amplitude for the outgoing scattering state is a $2 M \times 2 N$ matrix and reads

$\stackrel{A}{\underline{A}}^{\text {out }}(k)$

$$
=\left(\begin{array}{cccc}
t_{m_{L}}\left(\theta_{\alpha_{L L}}, k\right) & 0 & 0 & t_{m_{L}}\left(\theta_{\alpha_{L R}}, k\right) \\
0 & t_{m_{R}}\left(\theta_{\alpha_{R L}}, k\right) & t_{m_{R}}\left(\theta_{\alpha_{R R}}, k\right) & 0
\end{array}\right) .
$$

The $2 M \times 2 M$ dimensional $S$ matrix follows now from

$$
\underline{\underline{S}}^{\mathrm{PSC}}(k)=\underline{\underline{A}}^{\mathrm{out}}(k) \underline{\underline{G}}^{\mathrm{PSC}}(k) \underline{\underline{A}}^{\mathrm{in}}(k)=\left(\begin{array}{ll}
R_{m_{L}^{\prime}, m_{L}} & T_{m_{L}^{\prime}, m_{R}} \\
T_{m_{R}^{\prime}, m_{L}} & R_{m_{R}^{\prime}, m_{R}}
\end{array}\right) .
$$

With Eq. (4.9) the semiclassical calculation of the $S$ matrix of a hard-walled quantum billiard with two point contacts is reduced to a sequence of matrix multiplications. Systemspecific input are the data (length, angle of incidence and emission from point contact and Maslov index) of each of the four classes $(L \rightarrow L, L \rightarrow R, R \rightarrow L, R \rightarrow L)$ of classical trajectories.

\section{NUMERICAL RESULTS}

In the following we will apply the pseudopath semiclassical approximation to the calculation of transmission and reflection amplitudes in different regular and chaotic structures (i.e., circle, rectangle, and stadium). We compare the PSCA calculations with the results of the standard semiclassical approximation [10], exact quantum calculations [43], and microwave experiments [44].

In order to evaluate the scattering matrix elements $T_{m^{\prime}, m}$ and $R_{m^{\prime}, m}$ numerically we have used the truncated form of Eq. (4.5a) with $i \leqslant K$, where $K$ is the maximum number of diffractive scatterings (or "kinks"). We therefore relate $K$ to the maximum pathlength $l_{\max }$ of classical paths included. In order to include all pseudopaths with at least the same length, we require that the length of the shortest trajectory in the system $l_{\min }$ multiplied by $K+1$ has to be larger than $l_{\max }$ to guarantee that all pseudopaths up to $l_{\max }$ are included. Hence we require

$$
K \geqslant\left[\frac{l_{\max }}{l_{\min }}\right]-1,
$$

where the bracket stands for the largest integer less than the argument. This requirement only assure that each classical path is shadowed by a pseudopath of comparable length. The converse is evidently not the case. Pseudopaths are permitted with pathlength up to $l_{\max }(K+1)$ for which no classical counterpart of comparable length is included. We account for this deficiency by Fourier filtering the power spectra $P(l)$ of the

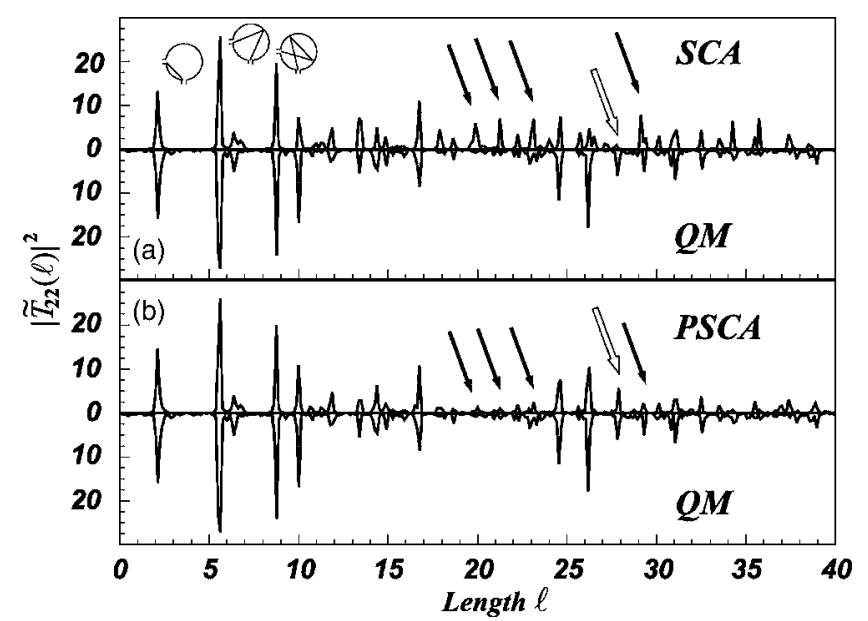

FIG. 4. Power spectra $\left|\widetilde{T}_{22}(l)\right|^{2}$ of the transmission amplitude in the circle billiard with perpendicular leads with $R=\sqrt{1+4 / \pi}$ and $d=0.25$ (see inset Fig. 5) for a finite window of $k, 1 \leqslant k \leqslant 6$ in units of $\pi / d$.

$S$-matrix elements for $l>l_{\max }$. The classical input data are determined analytically for the square and circle billiards while for the chaotic stadium billiards the data are generated by calculating the classical trajectories numerically.

\section{A. Regular structures}

For regular structures classical and pseudopaths can be easily enumerated and calculations up to very high path length $(l=40)$ have been performed as a sensitive test of semiclassical approximations. We present in the following results for the path-length power spectrum [Eq. (2.9)]. Figures 4 and 5 show the power spectra of the transmission and reflection amplitudes for the second mode, $T_{22}$ and $R_{22}$, in the circular billiard with perpendicular leads. Note that all the scattering geometries which we investigate in the following have the same cavity area $A=4+\pi$, or, accordingly, a linear dimension $D=\sqrt{4}+\pi \approx 2.7$.

Here we mainly concentrate on the improvement of the PSCA in comparison to the SCA. For $T_{22}$, the overestimation

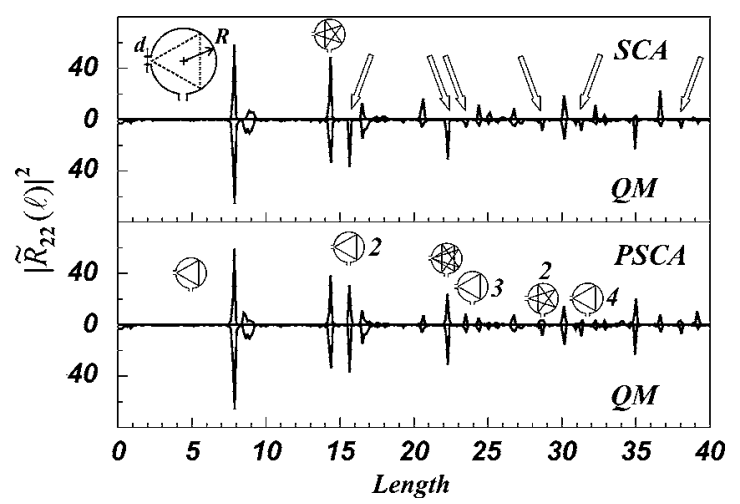

FIG. 5. Power spectra $\left|\widetilde{R}_{22}(l)\right|^{2}$ of the transmission amplitude in the circle billiard with perpendicular leads with $R=\sqrt{1+4 / \pi}$ and $d=0.25$ for a finite window of $k, 1 \leqslant k \leqslant 6$ in units of $\pi / d$. For more details see text. 
of long paths $(l>18)$ in the SCA calculation is clearly visible: compare the peaks labeled by black arrows in Fig. 4(a) with those in Fig. 4(b). The contributions of the additional pseudopaths included in the PSCA are responsible for the cancellation effects which suppress the peak heights. The peak heights of the PSCA agree well with the quantum calculations for long paths. Also the lack of individual peaks (i.e., pseudopaths) present in the quantum spectrum but missing in the SCA spectra (e.g., at $l \approx 27.5$; see white arrows in Fig. 4) can be addressed in the transmission amplitude.

This defect of the SCA can be seen more clearly in the plot for $R_{22}$ (see arrows in the upper part of Fig. 5). The pseudopath semiclassics provides for the additional peaks in agreement with the quantum calculation. The insets in the lower part of Fig. 5 schematically show the geometry of dominant pseudopaths, which experience at least one diffractive reflection at an open lead. The number next to the insets gives the information on how often the segment of a classical path has to be traversed to build up the corresponding pseudopath.

\section{B. Chaotic structures (the Bunimovich stadium)}

Up to now we have compared the SCA and PSCA for open billiards with regular classical dynamics. We turn now to chaotic (i.e., nonregular) billiards. The Bunimovich stadium $[38,45,46]$ serves as prototype system for structures with chaotic classical dynamics. Before we discuss our numerical results, we point out some of the characteristic differences between the regular and chaotic structures. In the stadium billiard the number of path bundles [10] up to a fixed length increases exponentially. The exponential proliferation represents a major challenge for the summation of paths. This leads to technical problems for the calculation of transport quantities in chaotic structures [38] due to the limitation of the number of paths that realistically can be taken into account. Furthermore, the path length distribution in chaotic structures differs qualitatively from that of regular structures. For the latter we find an algebraically decaying for the classical path-length distribution in contrast to the exponential decay for classically chaotic structures. This is to be distinguished from the exponential decay behavior for pseudopaths. Wirtz et al. [15] have shown that for the rectangular billiard the SCA leads to a linear (algebraic) decay of the path length power spectra, while the PSCA gives rise to an exponential decay when pseudopaths are included. However, in chaotic systems, already classical paths proliferate exponentially as a function of the path length and can account for exponential suppression of large path lengths. Therefore, the lack of pseudopaths which also proliferate exponentially is less dramatically felt than in regular systems where the exponential proliferation of pseudopaths competes with only linear proliferation of classical paths. This observation is key to the surprising findings in previous semiclassical calculations that the agreement between the semiclassical and the quantum pathlength spectrum is better for chaotic rather than for regular systems $[10,14,17,22,38]$. However, also in chaotic systems, the effects of diffractive pseudopaths can be clearly seen. Figure 6 shows the power spectra of

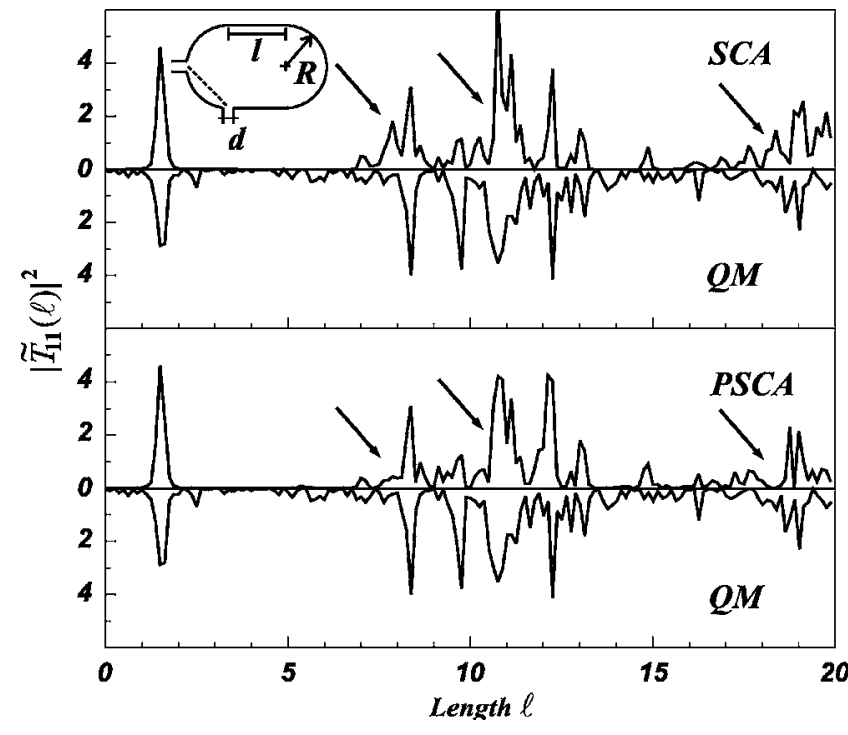

FIG. 6. Power spectra of the transmission amplitude $(m=n=1)$ in the stadium billiard with perpendicular leads with $R=1, l=2 R$, and $d=0.25$ for a finite window of $k, 0 \leqslant k \leqslant 5$ in units of $\pi / d$.

transmission amplitudes for the stadium with perpendicular leads. For $T_{11}$, remarkably, the path-length spectra within PSCA displays fewer pronounced peaks than the SCA in agreement with the quantum calculation. The reason is that in a chaotic system the high density of pseudopaths effectively causes path shadowing of true classical paths. As a result, some of the classical peaks are drastically reduced by destructive interference even for comparatively short path length.

\section{Comparison with microwave experiments}

As a third application we discuss the comparison between experimental studies of geometry-specific quantum scattering in microwave billiards [44] and semiclassical approximations (SCA and PSCA). The physics and modeling of microwave cavities are conceptually similar to that of semiconductor quantum dots due to the equivalence of the time-independent Schrödinger and Helmholtz equations [37]. Moreover, for microwave frequencies $\nu<\nu_{\max }=c / 2 h$ [37], where $h$ is the height of the microwave billiard, only a single transverse mode is supported by the cavity. This reduces the electromagnetic boundary conditions to Dirichlet-boundary conditions allowing for an exact correspondence between electrodynamics and quantum mechanics. Accordingly, the component of the electrical field perpendicular to the plane of the microwave billiard corresponds to the quantum mechanical wave function.

A direct measurement of transmission and reflection amplitudes (i.e., electrical field amplitudes) becomes possible. Moreover the semiclassical analysis presented above is directly applicable to describe scattering in microwave billiards.

The geometry of the microwave resonator used in the experiment [44] is shown as inset in Fig. 7. The transmission and reflection amplitude were taken in the frequency range 


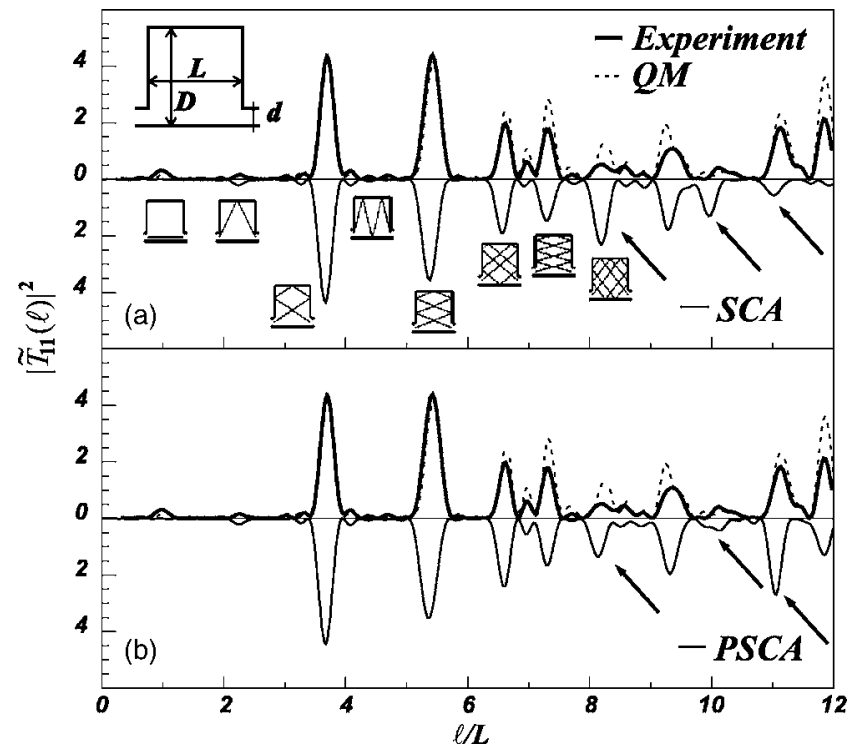

FIG. 7. Power spectra $\left|\widetilde{T}_{11}(l)\right|^{2}$ of the experimental and calculated (QM, SCA, and PSCA) transmission amplitude for the rectangular billiard $(L=225 \mathrm{~mm}, D=237 \mathrm{~mm}$, and $d=15.8 \mathrm{~mm}$ ) with opposite leads (not centered) for a finite window of $\nu$, $13 \mathrm{GHz} \leqslant \nu \leqslant 18 \mathrm{GHz}$. The characteristic peaks are identified in terms of classical transmitted trajectories (insets). Experimental data by Schanze [44].

$13 \mathrm{GHz} \leqslant \nu \leqslant 18 \mathrm{GHz}$, where only one mode is propagating in the waveguides. Figures 7 and 8 show the experimental and calculated data for the power spectra of the transmission and reflection amplitude, $\left|\widetilde{T}_{11}(l)\right|^{2}$ and $\left|\widetilde{R}_{11}(l)\right|^{2}$. The important role of pseudopaths in reproducing the correct peak pattern in good agreement with experimental results can be seen in both the transmission and the reflection spectra. Especially in $\left|\widetilde{R}_{11}(l)\right|^{2}$ distinct pseudopaths [see insets in Fig. 8(b)] ap-

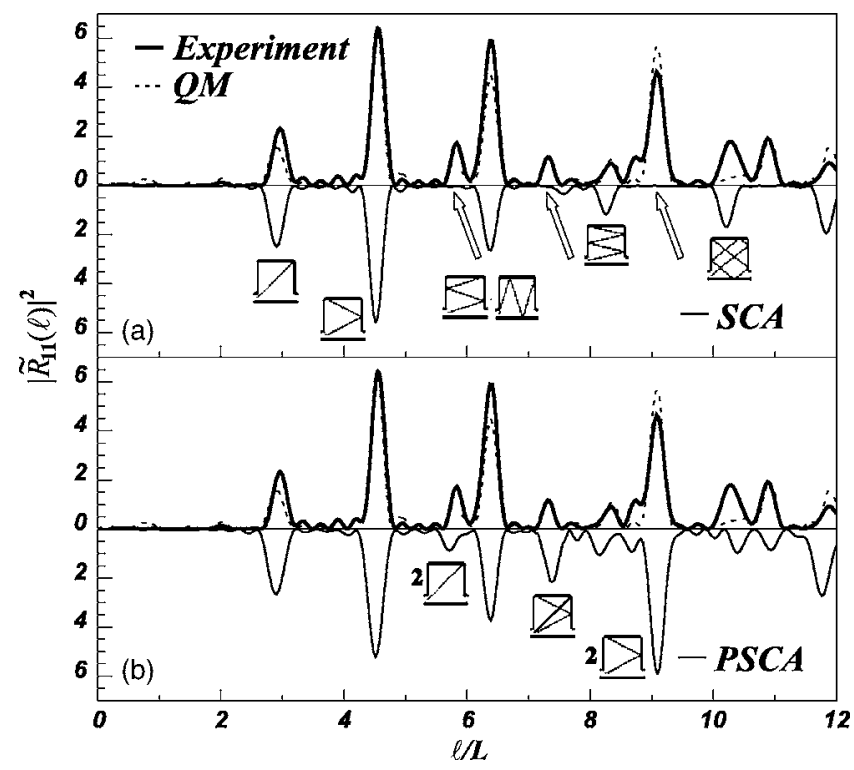

FIG. 8. Same as Fig. 7, but for reflection. The insets in (b) show the geometry of the pseudopaths. Numbers next to insets give the number of revolutions inside the billiard.
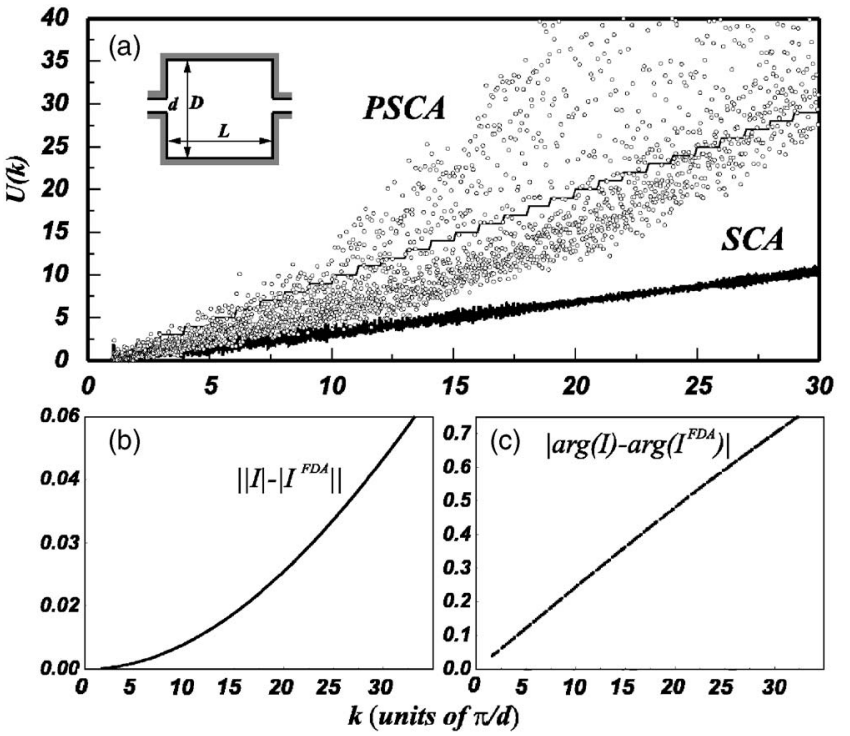

FIG. 9. (a) Test of unitarity $U(k)$ on different levels of semiclassical approximations $\left[\right.$ SCA and PSCA $\left.\left(K_{\max }=10\right)\right]$ to the rectangular billiard $(D=L=\sqrt{4+\pi}$ and $d=0.25)$. The staircase function shows the QM result where $U(k)=N(k)$ and $N(k)$ is the number of open modes. (b) shows the discrepancy between the numerical integration of $I$ and the FDA corresponding to Eq. (3.12) (solid line) and (c) the discrepancy in the phase angle.

pear which were noted in Ref. [44]. For example, the peak at $l \approx 9$ [right white arrow in Fig. 8(a)] is caused by the trajectory with the length $l \approx 4.5$ which, after one revolution in the billiard, is reflected back at the exit by the lead mouth, so that it continues for one more revolution. Thus its total length $l \approx 2 \times 4.5=9$. Of course, such nonclassical trajectories are not included in the standard semiclassical approximation [Fig. 8(a)]. In this special case, the reflection at the open lead mouth is specular or geometric. This peak would therefore also be present in the semiclassical approximation suggested by Schwieters et al. [7]. By contrast, nongeometric reflections ("kinks") by diffractive scattering are also present, e.g., the peak at $l=7.5$ [see inset in Fig. 8(b)]. The latter class is only contained in the PSCA.

\section{CONCLUSIONS}

We have presented an extension of the pseudopath semiclassical approximation (PSCA) [15] to billiards with arbitrary convex shape. A diagrammatic expansion of the semiclassical Dyson equation for the Green's function in terms of diffractive scattering diagrams is developed. The unperturbed Green's function represents the sum over classical paths. Each encounter with the lead (point contact) spawns new trajectories. By joining disjunct classical paths due to nongeometric (diffractive) scattering a large number of trajectories representing pseudopaths results. The Dyson integral equation can be converted to an algebraic matrix equation which can be solved by power series expansion or inversion. Using the examples of a circular and a stadium billiard we have shown numerically that the path-length power spectra calculated by the PSCA overcome shortcomings of standard 
semiclassical approximations by including an exponentially proliferating number of pseudopaths and converges towards quantum transport. Moreover, we have presented a comparison between microwave billiard experiments [44], the SCA and the PSCA calculation and find evidence for contributions by pseudopaths of PSCA to be present in the experimental data. We expect that our pseudopath semiclassical approximation will be able to address unresolved issues of semiclassical ballistic transport such as the problem of weak localization [16,39], the breakdown of symmetry of the autocorrelation function in reflection and transmission, and the semiclassical description of quantum shot noise [27].

\section{ACKNOWLEDGMENTS}

We thank H. Schanze, U. Kuhl, and H.-J. Stöckmann for providing the experimental data to allow a comparison between the microcavity experiment and our pseudopath semiclassical approximation. Support by the Grants Nos. FWFSFB016 and FWF-P17359 is gratefully acknowledged. L. W. acknowledges support by the European Community Network of Excellence NANOQUANTA (Grant No. NMP4-CT-2004500198).

\section{APPENDIX: FRAUNHOFER APPROXIMATION AND ITS LIMITATIONS}

The integrals along the transverse coordinate $(y)$ across the opening of the point contact are evaluated in Fraunhofer diffraction approximation. The dependence of the action in zero magnetic field $k L_{\alpha}(y)$ on the transverse coordinate $y$ is taken into account to first-order Taylor series expansion,

$$
L_{\alpha_{i}}(y)=L_{\alpha_{i}}+\sin \left(\theta_{\alpha_{i}}\right) y+\frac{\cos \left(\theta_{\alpha_{i}}\right)}{L_{\alpha_{i}}} y^{2} \cdots,
$$

where $L_{\alpha}=L_{\alpha}(y=0)$ is the length of the path that starts from the center of the lead mouth. Keeping all three terms in Eq. (A1) leads to Fresnel diffraction integrals. Dropping the third term results in a Fraunhofer diffraction approximation with the fundamental integral

$$
I^{\mathrm{FDA}}(\theta, n)=\frac{1}{\sqrt{2 d}} \int_{-d / 2}^{d / 2} e^{i\left(k_{n}+k \sin \theta\right) y} d y
$$

which, unlike Fresnel integrals, can be readily solved analytically in terms of elementary functions

$$
I^{\mathrm{FDA}}(\theta, n)=\sqrt{\frac{2}{d}}\left(\frac{\sin \left[\left(k_{n}^{i}+k \sin \theta\right) d / 2\right]}{k_{n}^{i}+k \sin \theta}\right) .
$$

In terms of Eq. (A3) the reflection amplitude [Eq. (3.12)] is given by

$$
r\left(\theta, \theta^{\prime}, k\right)=I^{\mathrm{FDA}}\left(\theta, \theta^{\prime},\right) k \sqrt{2 d\left(\cos \theta \cos \theta^{\prime}\right)},
$$

with $\theta^{\prime}=\sin ^{-1}\left(k_{n} / k\right)$, the transmission amplitude [Eq. (4.6)] by

$$
t_{m}(\theta, k)=\sqrt{k \cos \theta}\left[I^{\mathrm{FDA}}(\theta, m)+I^{\mathrm{FDA}}(\theta,-m)\right] .
$$

The validity of the FDA hinges on the condition that the third term in Eq. (A1) is negligible. This term is of the order $(k d)\left(d / L_{\alpha}\right) \leqq k d(d / D)$ for short paths. Since in quantum billiards the asymptotic far-field regime is never reached, the FDA, somewhat counterintuitively, will fail for large $k d$. Indeed, in Fig. 9(a) we show a test of unitarity $U(k)=R(k)+T(k)$ on different levels of semiclassical approximations to the rectangular billiard [inset of Fig. 9(a)] where the breakdown of the FDA and consequently of the PSCA for high modes $m>10$ (or $k d \geqslant 30$ ) can clearly be seen. To highlight the failure of the approximation used in Eq. (A1) or (3.12) we plotted in Figs. 9(b) and 9(c) (solid lines) the discrepancy between the exact solution of the integral $I$ [LHS of Eq. (3.12)] and the corresponding Fraunhofer approximation. For $L_{\alpha_{1}}=L_{\alpha_{2}}=L$ and $\theta_{\alpha_{1}}=\theta_{\alpha_{2}}=0$ as a function of $k$. Figure 9(b) shows the difference in the absolute values $|I|-\left|I^{\mathrm{FDA}}\right|$ and Fig. 9(c) the absolute discrepancy in the arguments $\left|\arg (I)-\arg \left(I^{\mathrm{FDA}}\right)\right|$. Since the phase discrepancy reaches a fraction of unity for $m \geqq 16$ [see Fig. 9(c)] random phases in the scattering amplitudes destroy path shadowing by pseudopaths and therefore cause the violation of unitarity [Fig. 9(a)]. The failure of the FDA has more dramatic consequences for the PSCA than for the SCA. This is due to the fact that in PSCA the pseudopath coupling leads to an exponentially growing number of FDAs with an increasing number of diffractive scatterings, in contrast to the linear scaling of the number of FDAs involved within SCA. The point to be stressed is that this failure is not due to the PSCA but due to the additional FDA. Applying more accurate diffraction integrals in this regime is expected to remedy the problem.
[1] M. C. Gutzwiller, Chaos in Classical and Quantum Mechanics (Springer Verlag, New York, 1991), and references therein.

[2] M. V. Berry and K. E. Mount, Rep. Prog. Phys. 35, 315 (1972).

[3] R. P. Feynman and A. R. Hibbs, Quantum Mechanics and Path Integrals (MacGraw-Hill, New York, 1965).

[4] S. Rotter, F. Libisch, J. Burgdörfer, U. Kuhl, and H.-J. Stöckmann, Phys. Rev. E 69, 046208 (2004).
[5] B. Weingartner, S. Rotter, and J. Burgdörfer, Phys. Rev. B (to be published).

[6] W. A. Lin, J. B. Delos, and R. V. Jensen, Chaos 3, 655 (1993).

[7] C. D. Schwieters, J. A. Alford, and J. B. Delos, Phys. Rev. B 54, 10652 (1996).

[8] E. Bogomolny, Nonlinearity 13, 947 (2000).

[9] X. Yang, H. Ishio, and J. Burgdörfer, Phys. Rev. B 52, 8219 (1994). 
[10] L. Wirtz, J.-Z. Tang, and J. Burgdörfer, Phys. Rev. B 56, 7589 (1997).

[11] L. Wirtz, J.-Z. Tang, and J. Burgdörfer, Phys. Rev. B 59, 2956 (1999).

[12] T. Blomquist and I. V. Zozoulenko, Phys. Rev. B 64, 195301 (2001); T. Blomquist and I. V. Zozoulenko, Phys. Scr. T90, 37 (2001).

[13] T. Blomquist, Phys. Rev. B 66, 155316 (2002).

[14] R. A. Jalabert, H. U. Baranger, and A. D. Stone, Phys. Rev. Lett. 65, 2442 (1990); H. U. Baranger, R. A. Jalabert, and A. D. Stone, Chaos 3, 665 (1993).

[15] L. Wirtz, C. Stampfer, S. Rotter, and J. Burgdörfer, Phys. Rev. E 67, 016206 (2003).

[16] H. U. Baranger, R. A. Jalabert, and A. D. Stone, Phys. Rev. Lett. 70, 3876 (1993).

[17] H. Ishio and J. Burgdörfer, Phys. Rev. B 51, 2013 (1995).

[18] G. Vattay, J. Cserti, G. Palla, and G. Szálka, Chaos, Solitons Fractals 8, 1031 (1997).

[19] I. V. Zozoulenko and T. Blomquist, Phys. Rev. B 67, 085320 (2003).

[20] R. Akis, D. K. Ferry, and J. P. Bird, Phys. Rev. Lett. 79, 123 (1997).

[21] E. Persson, I. Rotter, H.-J. Stöckmann, and M. Barth, Phys. Rev. Lett. 85, 2478 (2000).

[22] R. G. Nazmitdinov, K. N. Pichugin, I. Rotter, and P. Seba, Phys. Rev. E 64, 056214 (2001).

[23] R. G. Nazmitdinov, K. N. Pichugin, I. Rotter, and P. Seba, Phys. Rev. B 66, 085322 (2002).

[24] N. Argaman, Phys. Rev. Lett. 75, 2750 (1995); N. Argaman, Phys. Rev. B 53, 7035 (1995).

[25] K. Richter and M. Sieber, Phys. Rev. Lett. 89, 206801 (2002).

[26] R. S. Whitney and Ph. Jacquod, Phys. Rev. Lett. 94, 116801 (2005).

[27] Y. Blanter and M. Büttiker, Phys. Rep. 336, 1 (2000).

[28] S. Oberholzer, E. Sukhorukov, and C. Schönenberger, Nature
(London) 415, 765 (2002).

[29] C. W. J. Beenakker and Ch. Schönenberger, Phys. Today 56, 37 (2003).

[30] F. Aigner, S. Rotter, and J. Burgdörfer, Phys. Rev. Lett. 94, 216801 (2005).

[31] H. S. Sim and H. Schomerus, Phys. Rev. Lett. 89, 066801 (2002).

[32] P. G. Silvestrov, M. C. Goorden, and C. W. J. Beenakker, Phys. Rev. B 67, 241301(R) (2003).

[33] E. V. Sukhorukov and O. M. Bulalshenko, Phys. Rev. Lett. 94, 116803 (2005).

[34] Ph. Jacquod and E. V. Sukhorukov, Phys. Rev. Lett. 92, 116801 (2004).

[35] C. Marcus, A. J. Rimberg, R. M. Westervelt, P. F. Hopkins, and A. C. Gossard, Phys. Rev. Lett. 69, 506 (1992); Surf. Sci. 305, 480 (1994).

[36] M. W. Keller, O. Millo, A. Mittal, D. E. Prober, and R. N. Sacks, Surf. Sci. 305, 501 (1994).

[37] Y.-H. Kim, M. Barth, H.-J. Stöckmann, and J. P. Bird, Phys. Rev. B 65, 165317 (2002).

[38] S. Rotter, J.-Z. Tang, L. Wirtz, J. Trost, and J. Burgdörfer, Phys. Rev. B 62, 1950 (2000); S. Rotter, B. Weingartner, N. Rohringer, and J. Burgdörfer, ibid. 68, 165302 (2003).

[39] A. D. Stone, Les Houches Session LXI, edited by E. Akkermans et al. (North Holland, Amsterdam, 1995), p. 329.

[40] R. E. Prange, Phys. Rev. Lett. 90, 070401 (2003).

[41] P. Pichaureau and R. A. Jalabert, Eur. Phys. J. B 9, 299 (1999).

[42] R. Landauer, IBM J. Res. Dev. 1, 223 (1957).

[43] The quantum mechanical calculations were performed using the modular recursive Green's function method (MRGM) [38].

[44] T. Blomquist, H. Schanze, I. V. Zozoulenko, and H.-J. Stöckmann, Phys. Rev. E 66, 026217 (2002).

[45] G. Benettin and J. M. Strelcyn, Phys. Rev. A 17, 773 (1978).

[46] O. Bohigas, M. J. Giannoni, and C. Schmit, Phys. Rev. Lett. 52, 1 (1984). 